\title{
Spatial Pattern of Plant Specimen and Its Implications in Conservation Biology in Hengduan Mountains of Southwest China"
}

\author{
Hanming $\mathrm{He}^{1}$ and Jianmeng Feng ${ }^{2, * *}$ \\ ${ }^{1}$ Key Lab. of the Ministry of Education for Agro-Biodiversity and Pest Control, \\ Yunnan Agricultural University, Yunnan, Kunming 650201, China \\ ${ }^{2}$ Department of Life Science and Chemistry, Dali University, Dali 671000, China \\ fjmapku.org.cn
}

\begin{abstract}
In the past, the spatial patterns of specimen have not been paid enough attention. In this study, we probed the spatial pattern of plant specimen and its implications in conservation biology in Hengduan Mountains, one of the hotspots of global biodiversity, based on the dataset extracted from Chinese Virtual Herbarium. The results showed that there were big differences of specimen density among the units of the studied area. High specimen density was mainly found in the boundary regions of Northwest Yunnan, Southwest Sichuan and Southeast Tibet, the central part and the southern part of the studied area. The interest of the collectors or botanists was mainly focused on the units with low population density (low disturbance of human activity) and high complexity of topography. With the increase of specimen density, an increasing trend of species density was observed. Most of the units studied were not paid enough attention in the history of specimen collection. If we collected specimen at higher density in blank or marginalized area, we may have much more chances to find more taxonomies. The spatial pattern of specimen density may shape our understanding of the spatial pattern of specie diversity. We should not only inherit specimen from our predecessors, but also, we should probe into specimen repositories to understand biodiversity status and its spatial pattern.
\end{abstract}

Keywords: Altitudinal difference against area, Hengduan mountains, Population density, Spatial pattern, Specimen density, Species density, Vascular plants.

\section{Introduction}

Taxonomic records and their conservation are the basis of biodiversity research (Bickel, 1999; Ponder et al. 2001; Rudolf \& Torsren, 2004). With the accumulation of specimen in the long history of specimen collection, its implications in conservation biology are being paid much more attention in these days (Ponder et al. 2001). The inquiry into large specimen discussions can help us understand the characteristics and

\footnotetext{
This study was supported by Scientific Foundation of Yunnan (2010CD077).

*** Corresponding author.
} 
the spatial patterns of biodiversity in local or global scale (Chew \& Oheim, 2007; Greenstreet \& Piet, 2008; Rudolf \& Torsren, 2004). Also, it lends us a hand to detect the hotspots, in which lots of energy and time have been inputted in the past. Moreover, the probe into huge specimen discussions can help us to find or predict the blank or marginalized area neglected in the history of specimen collection more or less, and in which we may find more Taxonomies un-discovered in the past (Radim, 2007; Rudolf $\&$ Torsren, 2004). Therefore, the investigation on the spatial pattern of specimen and its implication in conservation biology can not be under-estimated.

Myers et al. (2000) published their research on biodiversity hotspots at global scale in Nature Magazine. The conclusions disclosed that Hengduan Mountains in Southwest China held high richness of biodiversity, and was one of 25 biodiversity hotspots in the world. Therefore, the study on the biodiversity in this region has drawn extensive and intensive attention (Sun, 2002). So, there was a long history of plant specimen collection, and a large amount of plant specimen from the studied area were accumulated $(\mathrm{Wu}$, 1988), which made the inquiry into the spatial pattern of plant specimen and its implications feasible. However, until now, no relevant study was reported.

Supported by China National Science \& Technology Infrastructure Center (SCNSTIC), Chinese Virtual Herbarium (CVH, www.cvh.org.cn) is aimed to provide essential and comprehensive information of plant specimen in China (Institute of Botany, Chinese Academy of Sciences, 2010). The herbarium covers most of plant specimen resources in China, with more than 2.5 million plant specimen digitalized and updated online. Until now, the herbarium is believed as the most complete database of plant specimen in China, serving us with taxonomic and distribution information of plant specimen across China with high reliability. So, our aim is to explore the spatial patterns of plant specimen and its implication in conservation biology in Hengduan Mountains, based on the dataset extracted from CVH. We hope this study can help us understand and protect plant diversity in the studied area. It must be noted that in this paper, we only focused the vascular plants in Hengduan Mountains.

\section{Data Collection and Methods}

All information of plant specimen in the studied area, including taxonomic and distribution information, was extracted from Chinese Virtual Herbarium. Then, we built a database of plant specimen of Hengduan Mountains, with more than 120,000 specimens and the information of plant specimen in each unit (county). Species richness in each unit was estimated from the specimen information. The topographical information and population density were from literatures (Zhao, 2005). To exclude the effects of area on the spatial pattern of specimen, we adopted specimen density to represent the amount of plant specimen in each unit. In the similar way, we adopted species density to stand for species richness (Logarithm transformation of area was conducted). In this paper, we adopted altitudinal difference against area (logarithm transformed) to represent topographical complexity, and the altitudinal difference was the difference between the highest altitude and lowest altitude in each unit studied.

Based on the dataset, dispersion coefficient was estimated to probe the differences of specimen density among 87 units studied. With the help of ArcGIS Desktop 8.3, we 
examined the spatial pattern of specimen density. To explain the pattern, Pearson's correlation between specimen density and population density, altitudinal difference against area was conducted. To check our hypothesis that the spatial pattern of specimen may shape our understanding of the patterns of species diversity, we conducted Pearson's correlation between specimen density and species density. Above analysis and drawings were completed with the help of ArcGIS Desktop 8.3, MS Excel and SPSS.

\section{Results}

\subsection{Frequency Distribution of Specimen Density and the Discreteness Analysis}

There were big differences of specimen density among 87 units studied (Fig.1). The lowest density was zero, while the highest density was 3.4 specimens per $\mathrm{km} 2$. There were 46 units (accounting for $52.9 \%$ of all units) whose specimen density was lower than 0.1 specimen per km2. Among 87 units studied, there were 78 units whose density was lower than 1 specimen per $\mathrm{km} 2$. It implied that most of the units displayed low specimen density, while high density was only observed in a small fraction of units studied. The discreteness analysis showed that the discreteness coefficient was 1.7, markedly higher than 1 , which meant that the distribution of specimen density among 87 units studied was extremely uneven.

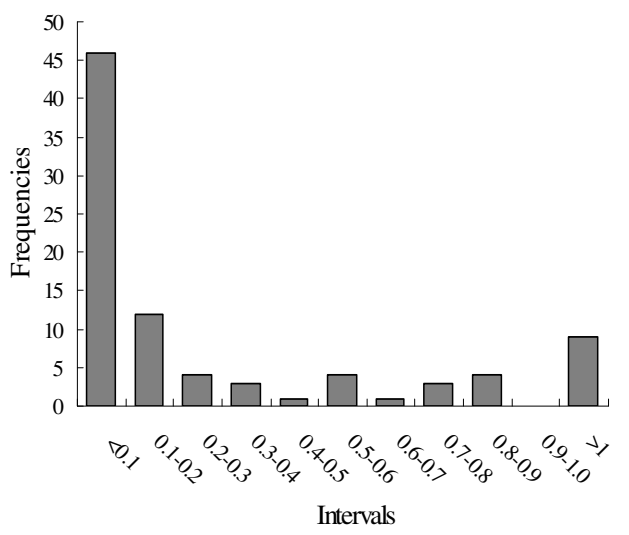

Fig. 1. Frequency distribution of specimen density in the units of Hengduan Mountains

\subsection{Spatial Pattern of Specimen Density and Its Interpretation}

High specimen density was observed in the boundary regions of the Northwest Yunnan, Southwest Sichuan and Southeast Tibet, and we also found the high density in the southern units and the central units of Hengduan Mountains (Fig.2). Except these units, low specimen density or blank units were detected in most of the units studied. Pearson's correlation indicated that with the increase of population density, there was a 
slight decreasing trend of specimen density among 85 units studied, except irregular data points of two units (Fig.3). There was strong correlation between specimen density and altitudinal difference against area (topographical complexity), and with the increase of the altitudinal difference against area, an increasing trend of specimen density can be predicted (Fig.4).

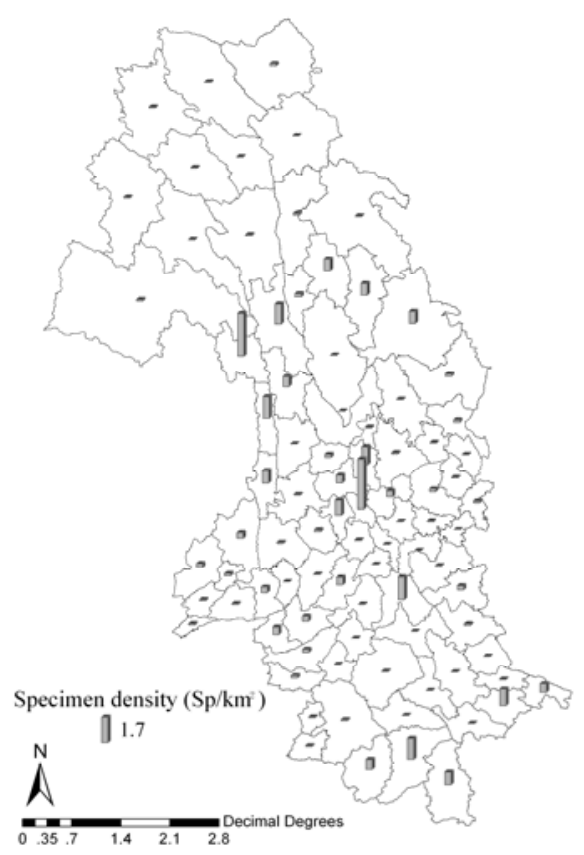

Fig. 2. Spatial patterns of specimen density of vascular plants in Hengduan Mountains

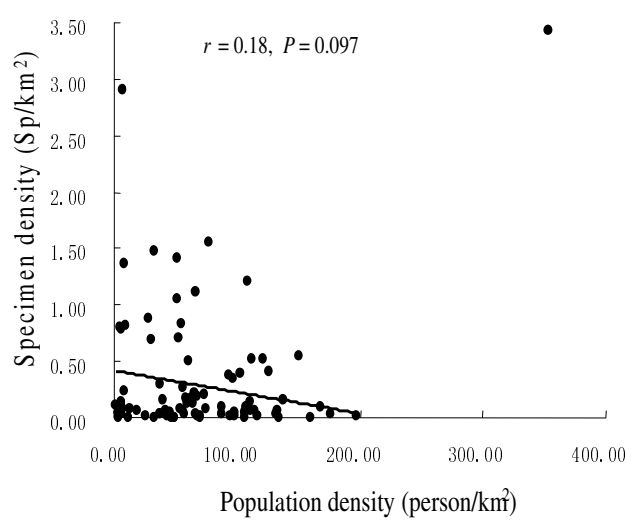

Fig. 3. Correlation between population density and specimen density 


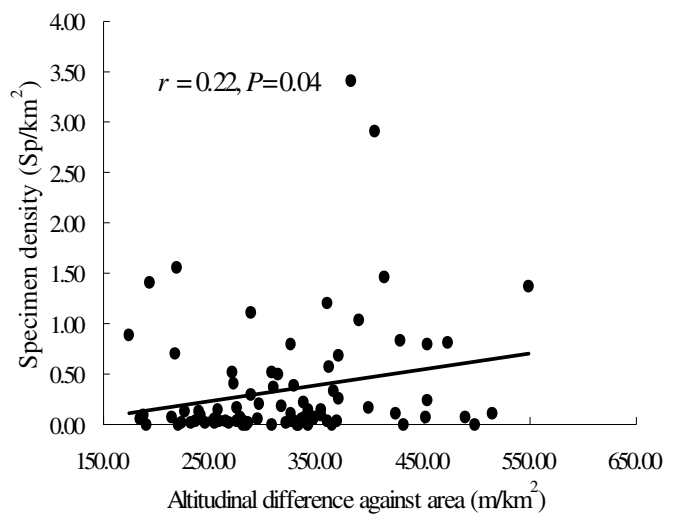

Fig. 4. Correlation between Altitudinal difference against area and specimen density

\subsection{Correlation between Specimen Density and Species Density}

We observed that there was strong correlation between specimen density and species density, and with the increase of specimen density, an increasing trend of species density can be perceived (Fig.5). It implied that in the units with lower specimen density, lower species density can be probably predicted, and vice versa.

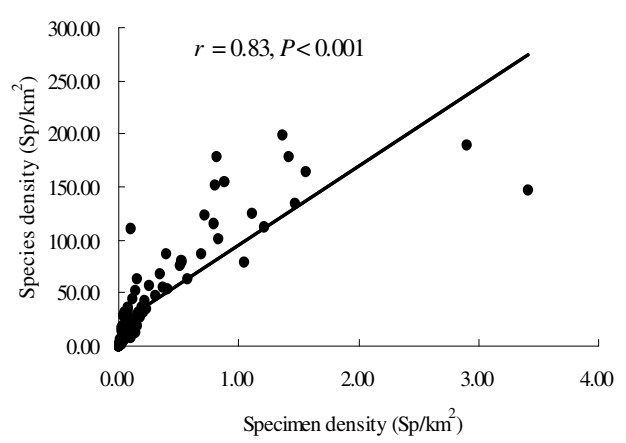

Fig. 5. Correlation between species density and specimen density

\subsection{The Implications in Conservation Biology}

The discreteness analysis indicated that the extreme unevenness of specimen density can be observed among the units studied, and the spatial pattern of specimen density also showed that the higher density was mainly found in the boundary regions of the three provinces, the central units and the south units of the studied area, which implied that these units were hotspots in the history of specimen collection and biodiversity research. However, in most of the units studied, the density was very low, and even some of them 
were blank area. So, we can infer that most of the units studied were paid little attention, or were overlooked in the history of specimen collection, more or less. The Pearson's correlation indicated that with the increase of altitudinal difference against area, an increasing trend of specimen density can be observed. Also, the results showed that there was slight negative correlation between population density and specimen density. It may imply that the interest of the collectors or botanists was mainly focused on the units with lower population density (low human disturbance) and higher complexity of topography, probably because raw or undisturbed vegetation and high richness of plant diversity in units with complex topography attracted their attention.

Previous study showed that if we conducted intensive specimen collection in the blank or marginalized regions, there were much more chances to find more taxonomies un-discovered in the regions in the past, compared with the attempt in biodiversity hotspots (Rudolf \& Torsren, 2004). In this paper, both discreteness analysis and the spatial pattern indicated that lots of blank or marginalized units were overlooked in the history of specimen collection. Therefore, we tentatively deduced that if we collected specimen at higher density in these blank or marginalized area, we may discover more species or taxonomies, which may enrich and refine our understanding of biodiversity in Hengduan Mountains and its spatial pattern.

In the results, we observed that there was strong correlation between species density and specimen density. It implied that higher intensity of specimen collection may enrich our findings in plant taxonomy in the studied area. It also indicated that specimen density would possibly affect our understanding of biodiversity in the studied area. If the intensity of specimen collection was low or not enough, we may underestimate the biodiversity in the studied area. However, if we conducted specimen collection at higher or enough intensity, we may find more species or taxonomies, and the results may more accurately reflect the biodiversity status in the studied area. Recently, Van Liefferinge (2010) observed that the investigation at higher sampling density may more reasonably reflect the biodiversity of fish in small brooks in Belgium. The studies in fungus also obtained similar conclusion (Whitcomb \& Stutz, 2007). Moreover, in the field of genetics diversity research, the studies testified that sampling density is an important factor affecting our understanding of genetics diversity (Alberton et al. 2006; Miyamoto et al. 2008; Muirhead et al. 2008). Other relevant studies also supported our conclusions (Chew \& Oheim, 2007; Greenstreet \& Piet, 2008). Therefore, the implications of the intensity of specimen collection can not be under-estimated.

The strong correlation between specimen density and species diversity also hinted that the spatial pattern of specimen density may shape our understanding of the spatial pattern of species richness. Recently, most of biodiversity research in the studied area focused on interpreting species richness patterns with climatic and topographical factors (Fu et al. 2007; Sherman et al. 2008; Yao et al. 2010; Zhang et al. 2009). But, our results implied that the spatial pattern of specimen density may be a strong potential factor, and may shape or even distort our understanding of spatial patterns of biodiversity. Considering the unevenness of the spatial pattern of specimen density and lots of blank or marginalized units, we tentatively assumed that we had not fully understood the spatial pattern of plant species diversity in Hengduan Mountains. Rodrigo \& Michael (2007) investigated the fern diversity in Bolivia, and found that 
sampling density strongly shaped their understanding of spatial patterns of fern diversity, which in some extents supported our conclusions.

The results showed that with the increasing of specimen density, the increasing trend of species density can be evidently observed. It may imply that the overall intensity of specimen collection was not enough. We should increase our intensity of specimen collection to enrich our understanding of plant diversity in the studied area. And it may also imply that the plant specimen in our dataset only represent a small fraction of plant species in the studied area. In 2004, Rudolf \& Torsren explored the species diversity of Euscelidia, and revealed that the specimen in museums only represented $50 \%$ of species in reality (Rudolf \& Torsren, 2004), which in some extents supported our results.

The specimen was important and fundamental resources in conservation biology, and we should input much energy and time on the collection, arrangement of specimen and the construction of specimen repository. However, in the past, the specimen's implications in conservation biology have not been paid enough attention in the studied area. In this paper, we suggested that we should not only inherit specimen from our predecessors. But also, we should probe into the specimen discussions to understand, assess the biodiversity status and its spatial pattern, and evaluate its hotspots and marginalized area. Furthermore, compared with other methods, the research based on specimen repository is one of the most efficient methods at the lowest costs (Rudolf \& Torsren, 2004), therefore, we should fully exploit the resources of specimen repository (Ponder et al., 2001; Rudolf \& Torsren, 2004).

\section{Conclusions}

In this study, we probed the spatial pattern of plant specimen and its implications in conservation biology in Hengduan Mountain. The results showed that there were big differences of specimen density among the units of the studied area. High specimen density was mainly found in the boundary regions of Northwest Yunnan, Southwest Sichuan and Southeast Tibet, the central part and the southern part of the studied area. The interest of the collectors or botanists was mainly focused on the units with low population density (low disturbance of human activity) and high complexity of topography. With the increase of specimen density, an increasing trend of species density was observed. Most of the units studied were not paid enough attention in the history of specimen collection.

\section{References}

1. Alberton, O., Kaschuk, G., Hungria, M.: Sampling effects on the assessment of genetic diversity of rhizobia associated with soybean and common bean. J. Soil Biol. Biochem. 38, 1298-1307 (2006)

2. Bickel, D.J.: What museum collections can reveal about species accumulation, richness, and rarity: an example from the Diptera. In: Ponder, W., Lunney, D. (eds.) The other 99\%: the Conservation and Biodiversity of Invertebrates. J. Royal Zoological Society of New South Wales, Mosman, Australia, Mosman, Australia, pp. 174-181 (1999) 
3. Chew, A., Oheim, K.: Teasing apart the effects of taphonomic and sampling bias on species diversity estimates using gis. J. Vertebr Paleontol. 27, 58A (2007)

4. Chinese Virtual Herbarium: Institute of Botany, Chinese Academy of Sciences, Beijing, http: / / www. cvh.org.cn (cited March 1, 2010)

5. Fu, C.Z., Wang, J.X., Pu, Z.C., Zhang, S.L., Chen, H.L., Zhao, B., Chen, J.K., Wu, J.H.: Elevational gradients of diversity for lizards and snakes in the Hengduan Mountains. J. China. Biodivers \& Conserv. 16(3), 707-726 (2007)

6. Greenstreet, S.P.R., Piet, G.J.: Assessing the sampling effort required to estimate alpha species diversity in the groundfish assemblages of the North Sea. J. Mar. Ecol.-Prog. Ser. 364, 181-197 (2008)

7. Miyamoto, N., Fernandez-Manjarres, J.F., Morand-Prieur, M.E., Bertolino, P., Frascaria-Lacoste, N.: What sampling is needed for reliable estimations of genetic diversity in Fraxinus excelsior L. J. Ann For Sci. 65, 403-410 (2008)

8. Muirhead, J.R., Gray, D.K., David, W.K., Sandra, M.E., Damiel, D.H., Hugh, J.M.: Identifying the source of species invasions: sampling intensity vs. J. Genetic Diversity. Mol. Ecol. 17, 1020-1035 (2008)

9. Myers, N., Mittermeier, R.A., Mittermeier, C.G., da Fonseca, G.A.B., Kent, J.: Biodiversity hotspots for conservation priorities. J. Nature 43, 853-858 (2000)

10. Ponder, W.F., Carter, G.A., Flemons, P., Chapman, R.R.: Evaluation of museum collection data for use in biodiversity assessment. J. Conserv. Biol. 15, 648-657 (2001)

11. Radim, H.: Is Sampling subjectivity a distorting factor in surveys for vegetation diversity? J. Folia Geobot. 42, 191-198 (2007)

12. Rudolf, M., Torsten, D.: Significance of Specimen Databases from Taxonomic Revisions for Estimating and Mapping the Global Species Diversity of Invertebrates and Repatriating Reliable Specimen Data. J. Conserv. Biol. 18, 478-488 (2004)

13. Sherman, R., Mullen, R., Haomin, L., Fang, Z.D., Wang, Y.: Spatial patterns of plant diversity and communities in Alpine ecosystems of the Hengduan Mountains, Northwest Yunnan, China. J. Plant Ecol-UK 1(2), 117-136 (2008)

14. Soria-Auza, R.W., Kessler, M.: The influence of sampling intensity on the perception of the spatial distribution of tropical diversity and endemism: a case study of ferns from Bolivia. J. Divers Distrib. 14(1), 123-130 (2007)

15. Sun, H.: Evolution of Arctic-Tertiary Xora in Himalayan-Hengduan Mountains. J. Acta Bot. Yunnan 24, 671-688 (2002)

16. Van Liefferinge, C., Simoens, I., Vogt, C., Cox, T.J.S., Breine, J., Ercken, D., Goethals, P., Claude, P.M.: Impact of habitat diversity on the sampling effort required for the assessment of river fish communities and IBI. Hydrobiologia 644, 169-183 (2010)

17. Whitcomb, S., Stutz, J.C.: Assessing diversity of arbuscular mycorrhizal fungi in a local community: role of sampling effort and spatial heterogeneity. J. Mycorrhiza 17, 429-437 (2007)

18. Wu, Z.Y.: The Hengduan Mountains flora and her significance. J. Jpn. Bot. 63(9), 1-14 (1988)

19. Yao, Y.H., Zhang, B.P., Han, F., Pang, Y.: Diversity and Geographical Pattern of Altitudinal Belts in the Hengduan Mountains in China. J. Mt Sci. 7(2), 123-132 (2010)

20. Zhang, D.C., Zhang, Y.H., Boufford, D.E., Sun, H.: Elevational patterns of species richness and endemism for some important taxa in the Hengduan Mountains, southwestern China. Biodivers. Conserv. 18(3), 699-716 (2009)

21. Zhao, D.H.: Album of Yunnan, 1st edn. Sinomap Press of China, Beijing (2005) 